\section{P426 RISK FACTORS ASSOCIATED WITH GONORRHEA INFECTION AMONG PREP PATIENTS IN A MONTREAL CLINIC}

${ }^{1}$ Joao Oliveira, ${ }^{2}$ Lorie Guibault, ${ }^{2}$ Judith Robin, ${ }^{2}$ Gabrielle Landry, ${ }^{2}$ Michel Boissonnault, ${ }^{2}$ Navid Niaki, ${ }^{2}$ Jason Szabo, ${ }^{2}$ Réjean Thomas*. 'Clinique Médicale I'Actuel, Epidemiology, Montreal, Canada; ${ }^{2}$ Clinique Médicale l'Actuel, Montreal, Canada

\subsection{6/sextrans-2019-sti.512}

Background Pre-exposure prophylaxis (PrEP) is effective for preventing HIV transmission. Studies demonstrated high sexually transmitted infection (STI) incidence among men who have sex with men (MSM) using PrEP. We aim to identify risk factors associated with Gonorrhea infection among our PrEP cohort from 2013 to 2018.

Methods This retrospective cohort study of the PrEP cohort of Clinique médicale l'Actuel (Montreal, Canada) including MSM, ages $\geq 18$, who consulted for PrEP from January 2013 to November 2018. We defined two groups: Gonorrhea infected (during 12 months after PrEP initiation) and noninfected. Cumulative incidence of Gonorrhea during 12 months after PrEP initiation was calculated. Behavioural risks characteristics (condom use, number of partners and history of STIs before PrEP) and Gonorrhea infection odds Ratio (OD) were compared between groups. Analyses were performed by using Stata 11.

Results Among 1159 patients who consulted for PrEP, 763 $(66 \%)$ had a university degree, $540(47 \%)$ an annual revenue higher than $\$ 55000$ and the median age was 36 years (IQR: 29-44); 929 (80\%) patients received daily baseline regimen. The Gonorrhea cumulative incidence was $18 \%$. There's no significant difference between groups, infected and non-infected respectively, in condomless anal sex $(95 \%$ v. $93 \%, \mathrm{p}=0.20)$ and mean number of sexual partner (19 vs 20, $\mathrm{p}=0.74)$. However, infected group reported history of STIs superior than non-infected $(84 \%$ vs $75 \%, p=0.006)$, translating to a Gonorrhea infection risk (OR: 1.77, 95\%CI: 1.18-2.64, adjusted by age, condomless anal sex and number of sexual partner).

Conclusion We found Gonorrhea infection among PrEP users was significantly associated with history of STIs. PrEP reaches high-risk individuals with STIs. Our results indicate the need for support which takes into consideration contextual lifestyle. The 3-month follow-up of patients on PrEP is a great opportunity to test and treat them frequently and to provide adequate and regular counseling.

Disclosure No significant relationships.

\section{P427 SUPPLEX: APPEARANCE AND PERFORMANCE- ENHANCING SUPPLEMENTS USE IN PEOPLE ON PRE-EXPOSURE PROPHYLAXIS}

${ }^{1}$ Salin Nhean, ${ }^{1}$ Alice Tseng, ${ }^{2}$ Nancy Sheehan, ${ }^{1}$ Isaac Bogoch*. ' University Health Network, Immunodeficiency Clinic, Toronto, Canada; ${ }^{2}$ McGlll University Health Centre, Chronic Viral IIIness Service, Montreal, Canada

\subsection{6/sextrans-2019-sti.513}

Background Men-who-have-sex-with-men (MSM) may experience more body image dissatisfaction compared to heterosexual men. The desire to be muscular has been linked with use of appearance- and performance-enhancing supplements (APES), including muscle-building supplements, steroids, and fat-burning products. APES use has been associated with potential health risks including serious liver and renal abnormalities. The purpose of this study was to describe the use and safety of APES among people attending an ambulatory pre-exposure prophylaxis (PrEP) clinic.

Methods All participants $\geq 18$ years on tenofovir disoproxil fumarate/emtricitabine were included; pregnant subjects were excluded. Prospective cross-sectional survey was conducted between February 1, 2018 and September 30, 2018 to assess APES usage, and retrospective chart reviews were done to determine the rates of liver and renal abnormalities from January 1, 2016 to September 30, 2018. RUCAM and Naranjo scales were used to assess causality with liver and renal abnormalities, respectively.

Results Among 50 participants (median 32 years, 52\% Caucasian, $86 \%$ MSM), 72\% reported lifetime APES use and 52\% with recent (within the past 6 months) use (APES group). Only 28\% had never used APES (non-APES group). APES and non-APES groups had similar rates of liver abnormalities (mostly Grade 1), but 2 (8\%) APES participants experienced Grade 3-4 elevations compared to none in the non-APES group. Liver enzyme elevations were possibly associated with creatine $(n=4)$, whey protein $(n=3)$, steroids $(n=2)$, and weight-loss supplements $(n=2)$. In the APES group, $12 \%$ had elevated serum creatinine (all stage 1) compared to none in the non-APES group. Whey protein $(n=2)$, creatine $(n=1)$, steroids $(n=1)$, and tenofovir disoproxil fumarate $(n=1)$ were possibly associated with renal abnormalities.

Conclusion APES usage among people on PrEP was high and possibly associated with liver and/or renal abnormalities. Increasing provider and consumer awareness of potential health risks of APES is encouraged to enhance safety.

Disclosure No significant relationships.

\section{P428 HIV INCIDENCE, AND PRE- AND POST-EXPOSURE PROPHYLAXIS (PREP AND PEP) AMONG PEP USERS AT NEW YORK CITY SEXUAL HEALTH CLINICS}

${ }^{1}$ Preeti Pathela*, ${ }^{2}$ Kelly Jamison, ${ }^{3}$ Sarah Braunstein, ${ }^{1}$ Christine Borges, ${ }^{3}$ Rachael Lazar, ${ }^{3}$ Demetre Daskalakis, 'Susan Blank. ${ }^{1}$ New York City Department of Health and Mental Hygiene, Bureau of Sexually Transmitted Infections, New York City, USA; ${ }^{2}$ New York City Department of Health and Mental Hygiene, Bureau of STI, New York City, USA; ${ }^{3}$ New York City Department of Health and Mental Hygiene, New York City, USA

\subsection{6/sextrans-2019-sti.514}

Background Sexually transmitted disease clinics are well-suited to deliver PEP; few provide full courses (28 days) due to cost. Since 2016, NYC Sexual Health Clinic (SHC) staff have provided full-course PEP to eligible HIV-negative patients, and thereafter attempt linkage to PrEP. We examined HIV incidence and patterns of PrEP/PEP use among a PEP cohort.

Methods We matched men-who-have-sex-with-men (MSM) who received PEP at SHC (09/2016-05/2017) to the citywide HIV surveillance registry to identify new HIV diagnoses between last PEP event during this interval and 06/30/2018. We calculated HIV incidence with time-at-risk starting 28 days after PEP provision; periods of additional PEP supplied at SHC during follow-up were excluded from time-at-risk. We examined subsequent PrEP and repeat PEP use during followup. For MSM without PrEP/repeat PEP, we calculated the number-needed-to-treat (NNT) with PrEP for one year to prevent 1 HIV infection (assumed 73\% efficacy with 90\% adherence). 